\title{
Damping of surface waves due to crude oil/oil emulsion films on water
}

\author{
Irina Sergievskaya ${ }^{\mathrm{a}}$, Stanislav Ermakov ${ }^{\mathrm{a}}$, Tatyana Lazareva ${ }^{\mathrm{a}}, \mathrm{Jie}_{\mathrm{G}} \mathrm{Guo}^{\mathrm{b}, \mathrm{c}, \mathrm{d}, *}$ \\ ${ }^{a}$ Institute of Applied Physics (IAP), Russian Academy of Sciences (RAS), Geophysical Research Division, 46 Ul'yanov Street, 603950 Nizhny Novgorod, Russia \\ ${ }^{\mathrm{b}}$ CAS Key Laboratory of Coastal Environmental Processes and Ecological Remediation, Yantai Institute of Coastal Zone Research (YIC), Chinese Academy of Sciences \\ (CAS), 17 Chunhui Road, Yantai 264003, PR China \\ c Shandong Key Laboratory of Coastal Environmental Processes, YICCAS, Yantai, Shandong 264003, PR China \\ ${ }^{\mathrm{d}}$ Center for Ocean Mega-Science, CAS, 7 Nanhai Road, Qingdao 266071, PR China
}

\section{A R T I C L E I N F O}

\section{Keywords:}

Crude oil

Oil emulsion

Wave damping

Laboratory experiment

\begin{abstract}
A B S T R A C T
In this paper results of laboratory studies of damping of gravity-capillary waves due to emulsified oil films (EOF) are presented and compared to crude oil films (COF). A laboratory method based on measuring the damping coefficient and the length of parametrically generated gravity-capillary waves is applied to a 50\% EOF and to crude oil films. Measurements of wave damping were carried out in a range of surface wave lengths, corresponding to Bragg waves of X- to Ka-band radars. The obtained dependences of wave damping coefficient on EOF thickness have demonstrated the existence of a damping maximum at thicknesses of about 1-2 mm, and the maximum is approximately twice the one for COF, the damping maximum for EOF is located at larger film thicknesses than for COF. Theoretical calculations of wave damping have been performed and viscoelastic parameters of EOF have been estimated from comparison between theory and experiment.
\end{abstract}

\section{Introduction}

It is well known that increased contamination of the ocean environment with hydrocarbons is associated with intense oil mining and transportation. The contamination can appear as oil films covering vast areas of the ocean surface. New opportunities of detection of oil pollutions using satellite radar/optical techniques are widely discussed in the literature (Alpers and Huehnerfuss, 1989; Ermakov et al., 1992; Scott and Thomas, 1999; Gade et al., 1998; Brekke and Solberg, 2005; Minchew et al., 2012; Migliaccio et al., 2015; Fingas and Brown, 2015; Fingas, 2018). Satellite methods of ocean remote sensing are based, in particular, on the surface roughness variations in the areas covered with mineral oil films - oil slicks. Oil slicks can be seen in microwave radar or/and optical images as dark or bright patches (the latter occur for optical observations at low grazing angles), because of two effects: suppression of wind waves and change of the reflection coefficient due to oil film. The first effect is usually more important, particularly for thin films which thickness is about $1 \mathrm{~mm}$ and less. Suppression of wind waves due to surface films is determined by their physical characteristics. The latter for monomolecular surfactant films are the surface tension and film elasticity (Levich, 1962; Lucassen-Reynders and Lucassen, 1970). Wave damping, as well as the surface tension and film elasticity for films of fatty acids/alcohols, and for some polymeric surfactants were investigated in detail in Dysthe et al. (1986),
Huehnerfuss et al. (1984), Hühnerfuss (1986), Scott and Stephens (1972), Pogorzelski et al. (1984) and Ermakov and Kijashko (2006). Mineral oil/oil products spilled on water form films of finite thickness and wave damping in the presence of such "thick" oil films is determined by a larger set of parameters, which are the volume viscosity, surface and interfacial tensions, elasticities and viscosity coefficients (Jenkins and Jakobs, 1997). Some results on wave attenuation due to mineral oil films and estimated film elasticity are described in Pogorzelski et al. (1984) and Ermakov et al. (2006). More in depth studies of wave damping due to crude oil films (COF) at different film thickness and wave frequencies were carried out in Ermakov et al. (1996), Henderson (1998), Ermakov et al. (2013), Sergievskaya et al. (2016), Sergievskaya et al. (2017), Rajan and Henderson (2017) and Sergievskaya and Ermakov (2017), and dependences of some physical characteristics of COF on oil viscosity, thickness, temperature etc. were investigated.

The studies (Ermakov and Kijashko, 2006; Ermakov et al., 2013; Sergievskaya et al., 2016; Sergievskaya et al., 2017; Sergievskaya and Ermakov, 2017) were conducted using an original method of parametric waves for measuring damping of capillary-gravity waves on the water surface covered with surface films (Ermakov et al., 1996; Ermakov and Kijashko, 2006). The method is based on the effect of parametric excitation of standing gravity-capillary waves in a vertically oscillating container, so-called Faraday ripples. This method gives

\footnotetext{
* Corresponding author at: Yantai Institute of Coastal Zone Research, Chinese Academy of Sciences, 17 Chunhui Road, Laishan District, Yantai, PR China.

E-mail address: jguo@yic.ac.cn (J. Guo).
} 
much more reliable results concerning the damping coefficient values since films are not distorted ("blown out") due to the Stokes drift that is usually a problem when measuring the damping for progressive surface waves (Scott and Stephens, 1972; Pogorzelski et al., 1984). It has been shown in Ermakov and Kijashko (2006) that the relative wave damping coefficient (i.e. the damping coefficient normalized by its value for a clean water surface) for films of fatty acids and alcohols (oleic acid, oleyl alcohol, dodecyl alcohol) as a function of surfactant concentration has a maximum in the vicinity of saturated monolayer concentrations, while for polymer films of (poly) oxyethylene glycol ("Emkarox") two maxima occur (Ermakov and Kijashko, 2006), see also Scott and Stephens (1972). Accordingly, the film elasticity retrieved when comparing theory and experiment was obtained as monotonically growing and tending to some limiting values for "oversaturated" films of oleic acid, oleyl alcohol and dodecyl alcohol. The elasticity for Emkarox polymer films was obtained having a maximum at intermediate concentration values and tending to small values at high surfactant concentrations. Later, wave damping due to films of crude oil and some oil derivatives was investigated mostly at small film thickness using the parametric method and new peculiarities of wave damping and surface tension were revealed (Ermakov et al., 2012). Namely, Ermakov et al. (2012) reported about the existence of a maximum of wave damping for crude oil, although the conclusion was based on rather limited volume of data.

Physical properties of EOF such as stability, viscosity etc. have been described in the literature (see, e.g. Fingas and Fieldhouse, 2011 and references therein). However, characteristics of EOF, particularly, film viscoelasticity, in application to the problem of oil slicks remote sensing, were investigated insufficiently to describe quantitatively wave damping in oil spills. These characteristics are extremely important in the context of the problem of wind waves damping in oil spills for correct interpretation of observations, including satellite remote sensing. In particular, information about physical parameters of films used in special remote sensing experiments is crucial for quantitative interpretation of the experiments and for elaboration of the models of radar and optical imaging of film slicks. In the most of experiments on oil slick remote sensing (see, e.g. Hansen et al., 2016 and cited papers) the authors operated with such "categories" of films as "plant oil", "oil emulsion", "crude oil", "biogenic" films. There is practically no physical meaning in this classification, since characteristics of films even within the same category can be strongly different, being determined not only by a chemical composition, but also by surfactant concentration or thickness (for monomolecular films) for oil/oil derivative films, the degree of emulsification, water temperature, volume viscosity, etc. Accordingly, suppression of wind waves and slick signatures cannot be characterized just by the nature of a film, and interpretation of experiments in terms of such film "categories" can be ambiguous.

This paper is focused on results of extended studies of wave damping due to EOF and comparative analysis of wave damping due to films of crude oil "Urals" and oil emulsions prepared from the crude oil in a wide film thickness range. The paper is organized as follows. First, a parametric wave method used for measuring waves damping and methodology of the measurements are described in Section 2. Experimental results on wave damping are presented in Section 3 for both bulk oil/oil emulsions and for COF/EOF. Results of theoretical (numerical) analysis of wave damping due to films of finite thickness and comparison between theory and experiment are given in Section 4. Results finally are summarized in Section 5.

\section{A parametric wave method and methodology of experiment}

Laboratory measurements of wave damping in the presence of COF and EOF were carried out using a method of parametrically generated waves (see, Fig. 1) developed in Ermakov and Kijashko (2006) and Ermakov et al. (1996).

The wave damping coefficient is proportional to the threshold acceleration $\Delta g$ for parametric generation of standing gravity-capillary waves (GCW) in a vertically vibrating container with water, so called Faraday ripples. The Faraday ripples are excited most effectively if the wave frequency $\omega$ is equal to half the vibration frequency $\omega_{v i b}$. The relation between $\Delta g$ and the damping coefficient can be obtained when analyzing the equation for oscillations $\zeta$ of the water surface under the conditions of the variable acceleration of gravity. This equation deduced from the basic equations and can be presented as following

$\frac{\mathrm{d}^{2} \zeta}{d t^{2}}+\gamma \frac{\mathrm{d} \zeta}{d t}+\omega^{2}\left(1+\frac{\Delta \omega}{\omega} \cos 2 \omega t\right) \zeta=0$,

where $\Delta \omega=\frac{\Delta g k \cdot \tanh (k \mathrm{~h})}{2 \omega}, \gamma$ denotes the damping coefficient of the oscillations of the water surface, $h$ is water depth. This oscillatory system is known to be unstable and having a growing solution if $\gamma<\frac{\Delta \omega}{2}$, while in the opposite case the perturbations of the water surface dissipate. When analyzing parametrically excited standing waves in a container one should also take into account that the condition $\left(\gamma<\frac{\Delta \omega}{2}\right)$ should be fulfilled for those standing wave modes for which the boundary conditions at the walls of the container are satisfied. For the case of onedimensional waves in a rectangular container the number of half-wave lengths must be equal to the container length. Assuming that the threshold acceleration amplitude $\Delta g$ is small compared to the acceleration of gravity $g$, the wave damping coefficient of parametric waves can be written as

$\gamma_{t o t}=\frac{\Delta g \cdot k \cdot \tanh (k h)}{4 \omega}$,

where $\omega=\omega_{v i b} / 2$ and $\mathrm{k}$ denote the frequency and the wavenumber of Faraday ripples, respectively. The $k$-values can be measured using photographs of Faraday ripples in the container.

The effective surface tension $\sigma_{\text {eff }}$ (EST) of water covered with film can be retrieved from the dispersion equation of linear GCW which reads

$\omega^{2}(k)=\left(g k+\frac{\sigma_{e f f}}{\rho} \cdot \mathrm{k}^{3}\right) \tanh (k h)$

Note that $\sigma_{e f f}$ depends in general on the surface tension coefficients of the "air-film" surface and of the "water-film" interface, as well as on a parameter $k d$ ( $d$ is the film thickness). For "thick" films ( $k d \gg 1) \sigma_{e f f}$ is close to the surface tension of the upper surface only, for a "thin" film $(k d \ll 1) \sigma_{\text {eff }}$ tends to a sum of the surface tension coefficients of both the surface and the interface. If there is no film on the water surface then the EST is simply equal to the surface tension of clean water. Note that the method is not applicable for the measuring of very large damping coefficient values. This occurs for films with extremely high viscoelasticity and/or for GCW of high enough frequencies, when $\Delta g \geq g$ and parametric waves cannot be excited.

The total damping coefficient $\gamma_{\text {tot }}$ can be written as a sum of two components, namely

$\gamma_{\text {tot }}=\gamma+\gamma_{\text {wall }}$

The first component, $\gamma$, is due to wave energy dissipation in the viscous boundary layers beneath the surface and the interface, and another, $\gamma_{\text {wall }}$, due to dissipation in the viscous boundary layers at the walls of the container. In Eq. (4) we omitted a component associated with effects of capillary meniscus on the walls (Miles, 1991) assuming them small. The component $\gamma_{\text {wall }}$ was theoretically analyzed in Henderson (1998). We consider a particular case of parametric standing waves in a rectangular container with sides $a$ and $b$. Standing GCW with wave crests parallel to side $a$ have wavenumbers $k_{n}=n \pi / b$ (the integer $n$ is the number of GCW half-wavelengths along the side b), and $\gamma_{\text {wall }}$ can be written in the following simplified form (cf., Ermakov and Kijashko, 2006)

$\gamma_{\text {wall }}=\sqrt{\frac{\omega\left(k_{\mathrm{T}}\right) \nu^{*}}{2}} \cdot \frac{2 b+a}{a b}$ 
(a)

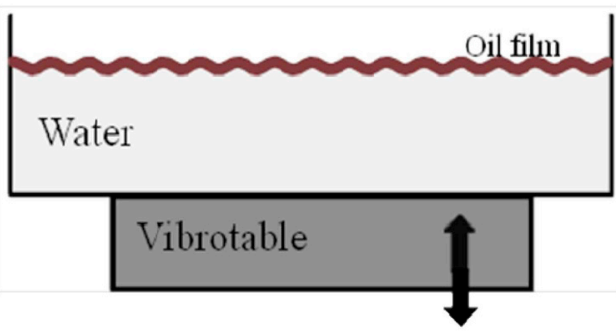

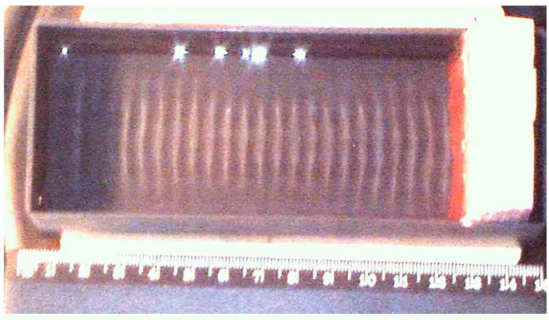

(b)

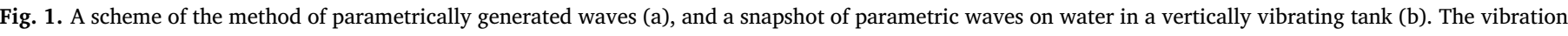
frequency is about $50 \mathrm{~Hz}$.

Here $\nu *$ is some effective viscosity of water with film. For the case of monomolecular films or films which thickness is small compared to the thickness of a viscous boundary layer the effective viscosity $\nu *$ is simply equal to the kinematic water viscosity $\nu$. In the opposite case when the film thickness is larger than the surface wave boundary layer, the effective viscosity $\nu *$ is close to the kinematic viscosity of bulk oil.

The amplitude wave damping coefficient $\gamma$ in the case of a very thin, e.g., monomolecular film of an insoluble surfactant can be written in the form (cf., e.g., Lucassen-Reynders and Lucassen, 1969)

$\gamma=2 \nu^{*} k^{2} \operatorname{Re}\left[\frac{1-X+X Y}{1-2 X+2 X^{2}}\right] X=\frac{E k^{2}}{\left(2 \omega^{3} \nu^{*} \rho^{2}\right)^{1 / 2}} Y=\frac{E k}{4 \rho \omega \nu^{*}}$

where $E=\Gamma \cdot \frac{\partial \sigma}{\partial \Gamma}, \Gamma$ is concentration of surfactants on the water surface, $\sigma$ is the surface tension coefficient, pis the fluid density. Eq. (6) can be used for films which thickness $d$ is small compared to the thickness of an oscillatory wave boundary layer that is $\mathrm{d}<<\sqrt{\nu / \omega}$.

When measuring the threshold acceleration $\Delta g$ at a given $k_{n}$ one that is able to retrieve $\gamma$ and the film elasticity from Eqs. (2)-(6). Note, however, that Eq. (6) can be satisfied in general for two E-values. The way to remove this uncertainty is to measure the damping coefficient at several (at least, two) wavenumbers and to compare with theory. One should emphasize that measurements at several wavenumbers are needed for the elasticity retrieval in the case of soluble surfactant films. Practically, however, measurements as several frequencies may not be useful if the error of measurements is rather high.

Measurements of wave damping were carried out with a small rectangular container of $3.8 \mathrm{~cm}$ in width, $11.8 \mathrm{~cm}$ in length and $4 \mathrm{~cm}$ in height. The container was mounted on a vibration table oscillating at selected frequencies in the range from about 20 to $50 \mathrm{~Hz}$ (see Fig. 1). Each of the frequencies was chosen from the condition that a ratio of the container length to the half wavelength is an integer and fine frequency tuning was then made to find a minimum threshold excitation value. The tuning is needed because the effective length of the container which determines $k_{n}$ is a little less than the geometric length because of meniscus.

Highly purified water passed through a set of membrane and carbon filters was used for the experiments. The water quality was checked when measuring the wave damping coefficient at several wavelengths. From the measured damping coefficient the water viscosity was retrieved when compared to theory. The water was considered as clean if the retrieved surface tension was close enough (within 15-20\% accuracy) to its table value. To create oil/oil emulsion films of a given thickness some known amounts of the substances were dropped on the water surface. One should emphasize, however, that the film thickness evaluated simply as a ratio of the volume of spilled surfactants to the water surface area is in fact larger than the real thickness. This is mostly because some uncontrolled amount of the substance is contained within the wall capillary meniscus, which volume is difficult to estimate precisely.

In the laboratory experiments we used oil which was a mixture of Urals and Siberian Light (the density of $860-871 \mathrm{~kg} / \mathrm{m}^{3}$ and $845-850 \mathrm{~kg} / \mathrm{m}^{3}$, respectively) which was provided by the staff of the
Kstovo oil refinery (Nizhny Novgorod region, Russia). The COF or EOF of different thicknesses was created on the water surface in the experimental container in two ways. The first one was to drop a given amount using a calibrated pipette to produce a film of a given thickness. To produce a film of another thickness the procedure was repeated from the beginning with another amount of oil. The second way was to carry out measurements at one thickness, then to do measurements after addition of a calibrated amount to the previously created film, etc. We, however, did not notice any significant difference between these two experimental procedures. All the experiments were carried out at the temperature of $24^{\circ} \mathrm{C}-25^{\circ} \mathrm{C}$. The $50 \%$-emulsions were prepared from the crude oil using an ultrasonic dispenser with cooling, and could be characterized as a "chocolate mousse". The emulsion and crude oil were stored in hermetic tanks and were spread on the water surface just before experiment. The emulsion was quite stable and its properties practically were not changed after a month or even longer, so one could consider the emulsion characteristics unchanged during the period of the experiments.

\section{Results}

\subsection{Damping of gravity-capillary waves on the surface of bulk crude oil/oil emulsion}

Let us present results on damping of gravity-capillary waves on the surfaces of crude oil and crude oil emulsion. The wave damping coefficient values with removed damping due to walls and divided by $2 k^{2}$ are shown in Fig. 2a in a wavenumber range from about 3 to $8 \mathrm{rad} / \mathrm{cm}$. Thus defined "effective" viscosity for pure water is presented for comparison, too. The "effective" viscosity values for pure water within the error of measurements are well consistent with table values of kinematic viscosity of pure water at room temperatures, i.e., $0.009-0.01 \mathrm{~cm}^{2} / \mathrm{s}$. The "effective" viscosity of crude oil and oil emulsions are at least one order of magnitude greater than the pure water viscosity, besides the "effective" viscosity of the oil emulsion is 2-3 times larger than those for crude oil at the low wavenumbers of about $3 \mathrm{rad} / \mathrm{cm}$.

The surface tension coefficient (see, Fig. 2b), which we further refer to an "effective surface tension", $\sigma_{\text {eff }}$, was retrieved from the dispersion relation of gravity-capillary waves $\sigma_{e f f}=\frac{\omega^{2}(k) / \tanh (k d)-g k}{\mathrm{k}^{3} / p}$ when measuring the frequency and wavenumber of parametrically generated waves. The "effective surface tension", EST, is practically constant for pure water and is very close to table values of the surface tension for pure water. For bulk crude oil and oil emulsion one can expect that $\sigma_{\text {eff }}$ should be constant, too, although this is only roughly valid (see, Fig. $2 \mathrm{~b}$ ), the mean $\sigma_{\text {eff }}$-values are about $32-34 \mathrm{mN} / \mathrm{m}$ for both the substances.

3.2. Wave damping in the presence of films of crude oil and oil emulsion on the water surface

Let us now present results of parametric wave measurements for the 

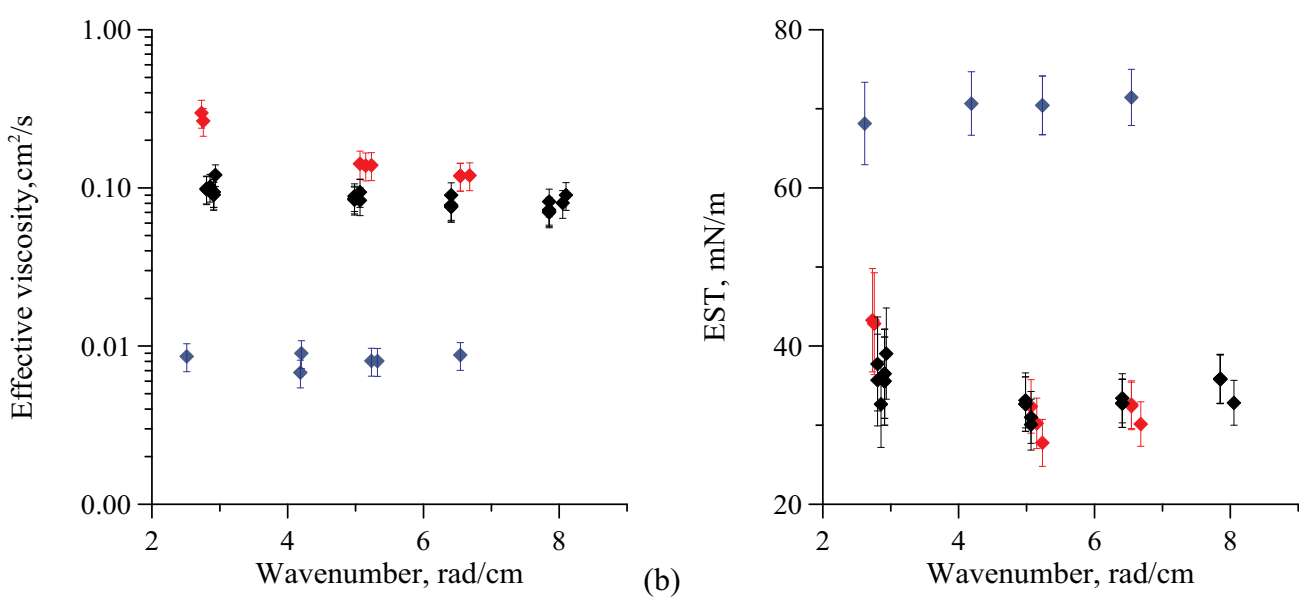

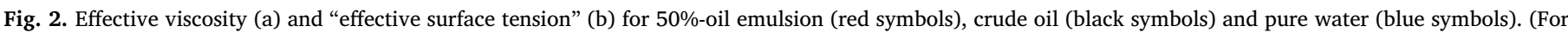
interpretation of the references to color in this figure legend, the reader is referred to the web version of this article.)

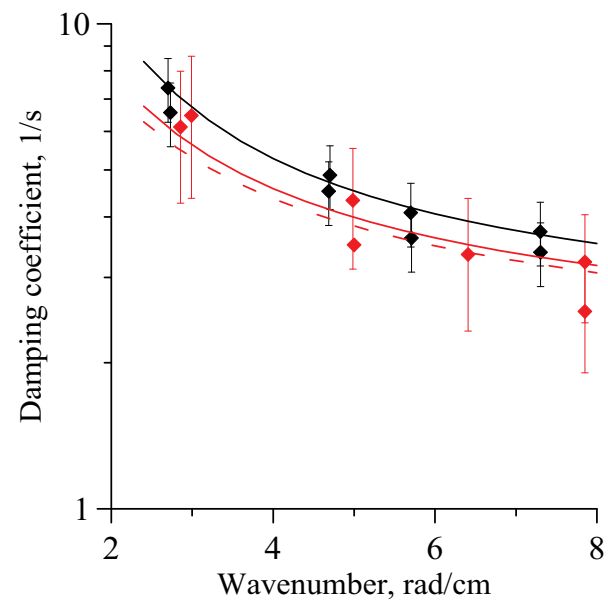

(b)

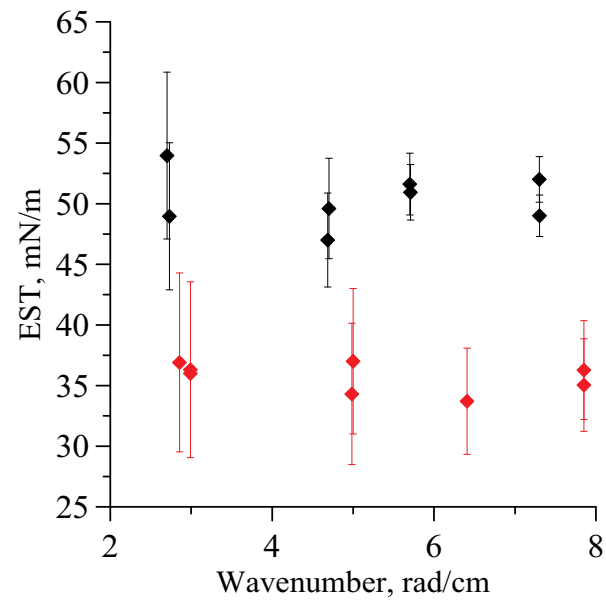

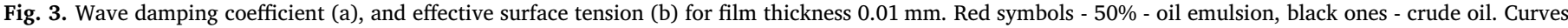

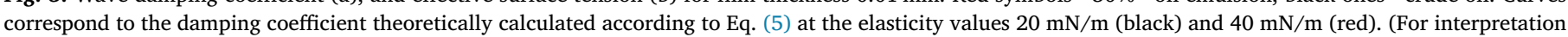
of the references to color in this figure legend, the reader is referred to the web version of this article.)

cases of films of crude oil/oil emulsion on water. At small film thicknesses the damping coefficient and the effective surface tension are shown in Fig. 3. It is worth noting that the damping coefficient decreases with wavenumber in the range from about $3 \mathrm{rad} / \mathrm{cm}$ to $8 \mathrm{rad} /$ $\mathrm{cm}$, and its values are not strongly different for COF and EOF. The surface tension is nearly independent on wavenumber, and the surface tensions for thin COF and EOF in Fig. 3b are different from each other, for EOF the surface tension drops stronger than for COF unlike the case of bulk oil and oil emulsion. Note, that both surface tensions in Fig. 3b are larger than for the bulk liquids, particularly for COF.

The most interesting results concern the dependences of wave damping and EST on film thickness (see, Fig. 4 for COF and Fig. 5 for EOF for the case of wave frequency of $10 \mathrm{~Hz}$ ). The damping coefficient achieves a maximum at the film thickness values of about $0.8 \mathrm{~mm}$ for $\mathrm{COF}$ and about $1.5 \mathrm{~mm}$ for EOF. The damping coefficient maximum for oil emulsions is about twice the one for COF. At the thicknesses corresponding to the damping maximum, the EST dependences demonstrate more complicated "zigzag" behavior, namely, the occurrence of a local minimum and an adjoining maximum both for EOF and COF. One should emphasize that the general behavior of the dependences of wave damping on film thickness is similar at different wave frequencies (see, in particular, our previous measurements for oil films by Ermakov et al., 2012).

Note that films with thickness comparable to or larger than the wave lengths can be considered practically as bulk substances. This is because the orbital motion in surface waves is limited by a surface layer with thicknesses of about wave length. As such, the relative damping coefficient in the considered wavenumber range for EOF/COF of 5-10$\mathrm{mm}$ thickness and larger should be consistent with the damping for the bulk crude oil and oil emulsion. Similarly, EST at large film thickness of COF and EOF should be close to the values shown in Fig. 2.

\section{Theory}

The damping of short gravity-capillary waves was investigated in the frame of a two-layer fluid model. The system consists of an upper layer of Newtonian viscous fluid of finite thickness floating on water of infinite depth (the latter assumption is taken for simplicity and is valid for the studied wave frequency range). The system is described by the continuity equation and linearized Navier-Stokes equations

$U_{ \pm x}+W_{ \pm z}=0$

$U_{ \pm t}+p_{ \pm x} / \rho_{ \pm}=v_{ \pm} \nabla^{2} U_{ \pm}$

$\mathrm{W}_{ \pm t}+p_{ \pm z} / \rho_{ \pm}+g z=\nu_{ \pm} \nabla^{2} W_{ \pm}$,

respectively.

The kinematic and dynamic boundary conditions at the upper film surface $\mathrm{z}=\mathrm{d}$ are 


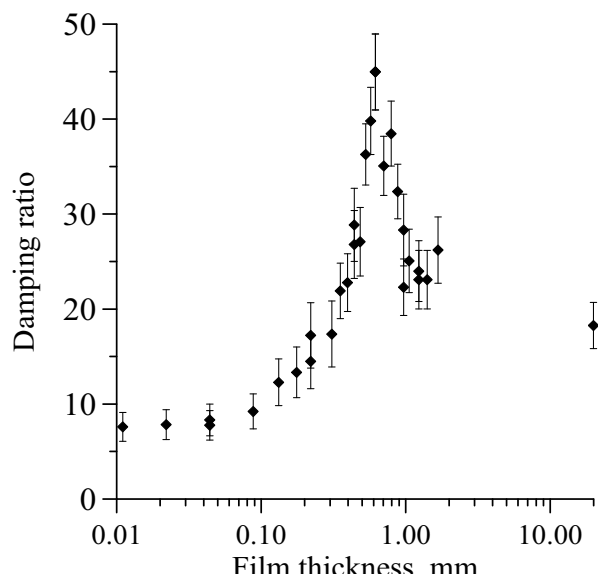

(a)
Film thickness, $\mathrm{mm}$

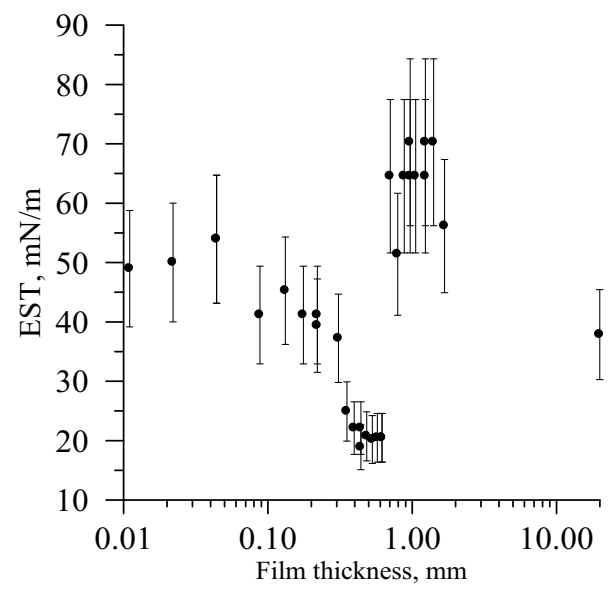

Fig. 4. Wave damping coefficient normalized by its value for clean water (damping ratio), and EST vs. film thickness for COF. Wave frequency is $10 \mathrm{~Hz}$.

$U_{+}=\xi_{+t}, W_{+}=\zeta_{+t}$

$\rho_{+} \nu_{+}\left(U_{+z}+W_{+x}\right)=E_{+} \xi_{x x}$

$p_{+}-2 v_{+} W_{+z}+\left(\sigma_{+} / \rho_{+}\right) \zeta_{+x x}=0$.

and at the oil/water interface $\mathrm{z}=0$ are (see, e.g. Jenkins and Jakobs, 1997)

$W_{+}=W_{-}=\zeta_{-t}, \quad U_{+}=U_{-}=\xi_{-t}$,

$\rho_{+} \nu_{+}\left(U_{+z}+W_{+x}\right)+E_{-} \xi_{x x}=\rho_{-} \nu_{-}\left(U_{-z}+W_{-z}\right)$,

$\rho_{+}\left(p_{+}-2 v_{+} W_{+z}\right)=\rho_{-}\left(p_{-}-2 v_{-} W_{-z}\right)+\sigma_{-} \zeta_{-x x}$.

In Eqs. (7)-(15) $W_{ \pm}$and $U_{ \pm}$denote vertical and horizontal wave orbital velocities, respectively, $p_{ \pm}$is pressure, the subindexes correspond to the upper $(+)$ and lower $(-)$ fluids, $\varsigma_{ \pm}$and $\xi_{ \pm}$are vertical and horizontal displacements of liquid particles, respectively, at the upper surface $(+)$ and at the interface $(-), g$ is acceleration of gravity, $\rho$ is fluid density, $\nu_{ \pm}$is kinematic viscosity, $\sigma_{ \pm}$is surface tension, $E_{ \pm}$ is elasticity of films on the upper surface and on the interface, low indexes $z, x, t$ denote partial derivatives for corresponding variables.

The system (Eqs. (7)-(15)) contains several physical parameters: kinematic viscosity of material of the upper and lower layers, film thickness $d$, the surface tension and elasticity coefficients for the free upper surface and for the interface. In general, the elasticity coefficients can be complex, e.g. for the case of a soluble film substance, thus depending on wave frequency. In our calculations below, however, the

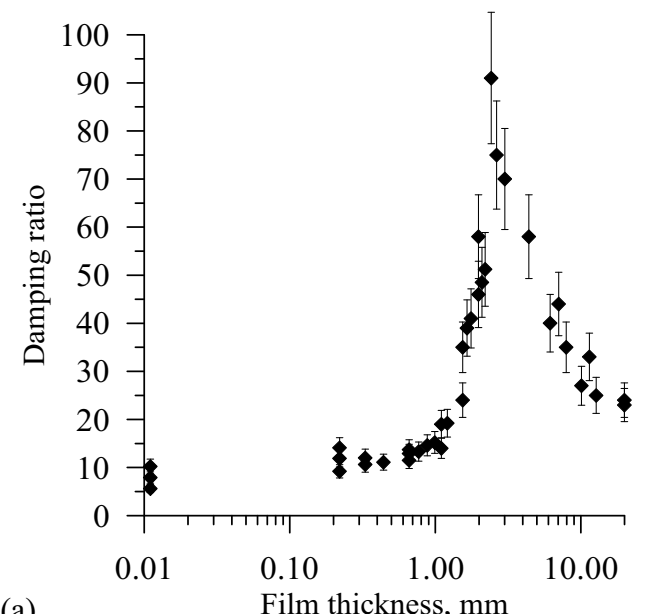

(a) elasticities are assumed as constant values.

The solution of Eqs. (7)-(15) was seeking in the form of progressive surface waves, propagating along the $\mathrm{x}$-axis

$\left(U_{ \pm}, W_{ \pm}, \ldots.\right) \propto\left(u_{ \pm}(z), w_{ \pm}(z), \ldots ..\right) \cdot e^{i k x-i \omega t}$

The wavenumber in Eq. (16) is considered as a real value, while the frequency $\omega$ is complex which imaginary part is equal to the wave damping coefficient. The vertical structure of the wave variables, e.g., for vertical velocity component, has the following form

$w_{ \pm}=c_{1 \pm} e^{k z}+c_{2 \pm} e^{-k z}+c_{3 \pm} e^{l_{ \pm} z}+c_{4 \pm} e^{-l_{ \pm} z}, \quad l_{\mp}=\sqrt{\frac{-i \omega}{v_{\mp}}+\mathrm{k}^{2}}$

The dispersion relation relating the frequency and wavenumber of surface waves can be obtained from Eqs. (7)-(17) in the form of the 8-th order determinant equal to zero, when seeking a nontrivial solution for the coefficients $c_{i \pm}$. Some results of numerical calculations are shown in Fig. 6, where the relative wave damping coefficient, i.e. the damping coefficient normalized by its value for the case of pure water, and the effective surface tension values are shown as functions of oil film thickness as some selected parameters of the upper and lower fluids. Fig. 6 shows that the damping coefficient can have a maximum over a wide range of oil thickness $(0.1 \mathrm{~mm}$ to $1 \mathrm{~mm})$ and varying elasticities from 10 to $40 \mathrm{mN} / \mathrm{m}$. In the same range the EST values demonstrate zigzag behavior. The mentioned peculiarities are qualitatively consistent with experiment, and more detailed analysis will be given in the next section.

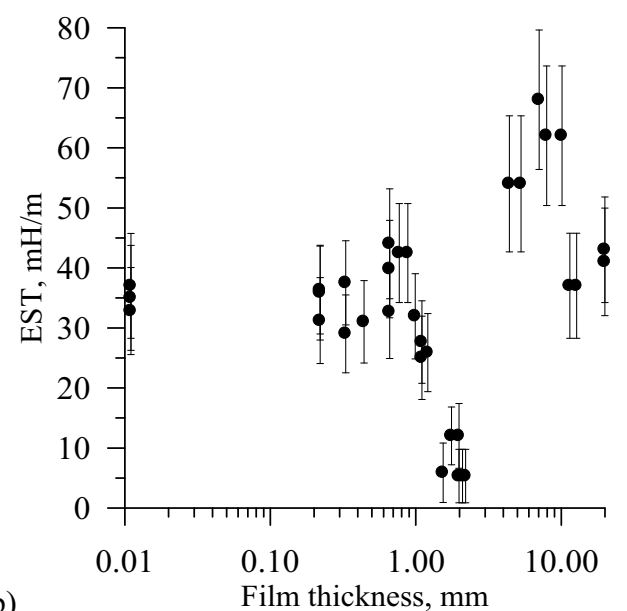

(b)

Film thickness, mm

Fig. 5. Same as in Fig. 4 for oil emulsion films. 


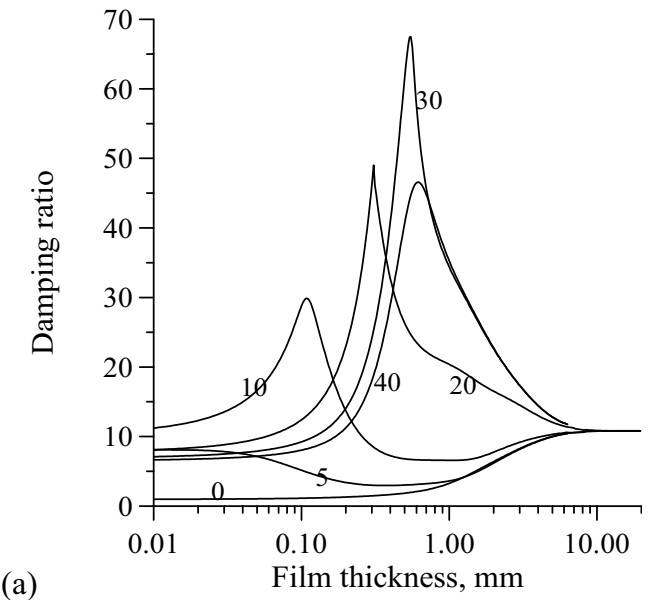

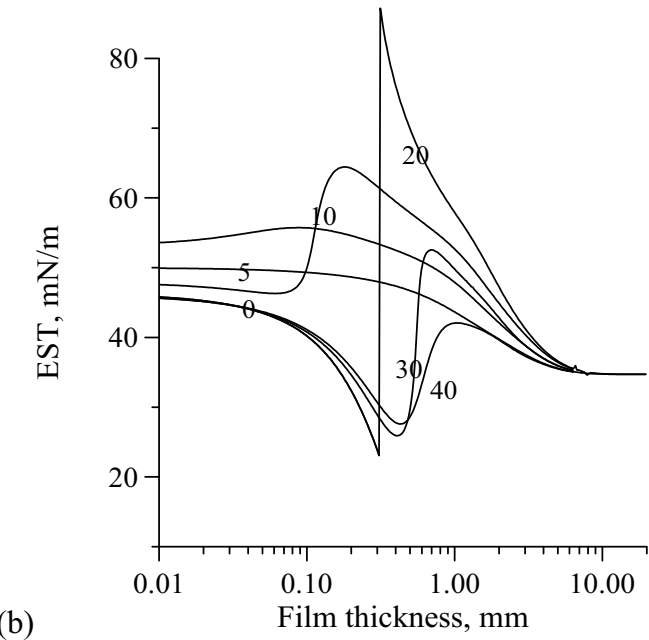

(b)

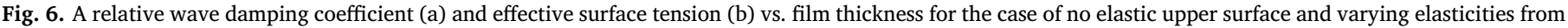
5 to $40 \mathrm{mN} / \mathrm{m}$. Wave frequency of $10 \mathrm{~Hz}, \nu_{+}=0.09 \mathrm{~cm}^{2} / \mathrm{s}, \rho_{+}=0.85 \mathrm{~g} / \mathrm{cm}^{3}, \rho_{-}=1 \mathrm{~g} / \mathrm{cm}^{3}, \nu_{-}=0.0085 \mathrm{~cm}{ }^{2} / \mathrm{s}, \sigma_{+}=30 \mathrm{mN} / \mathrm{m}, \sigma_{-}=20 \mathrm{mN} / \mathrm{m}, E_{+}=0$.

\section{Discussion}

\subsection{Gravity-capillary waves on the surface of bulk crude oil/oil emulsion}

Let us discuss first the results on wave damping and surface tension for bulk crude oil and oil emulsions. The obtained values of the "effective viscosity" $\nu_{\text {eff }}=\gamma / 2 k^{2}$ are shown in Fig. 7. The "effective viscosity" for pure water in Fig. 7 is practically constant and the measured viscosity values about $0.009 \mathrm{~cm}^{2} / \mathrm{s}$ are well consistent with the table kinematic water viscosity values at room temperatures of $25^{\circ} \mathrm{C}$.

The "effective viscosity" for crude oil, although being slightly decreasing with wavenumber, can be nevertheless considered within the error of measurements as a constant value close to $0.09 \mathrm{~cm}^{2} / \mathrm{c}$. On the contrary, the decrease of the "effective viscosity" with wavenumber for EOF is larger than the error of experiment and cannot be ignored. This result can be explained under the assumption that a thin (very likely, quasi monomolecular) film is formed on the surface of the oil emulsion layer. This film appears due to accumulation of the "third", most surface-active agent which is responsible for oil emulsion stability and which comes from the oil fraction decreasing the interface tension between water and oil fractions (see, e.g. Adamson, 1982). The

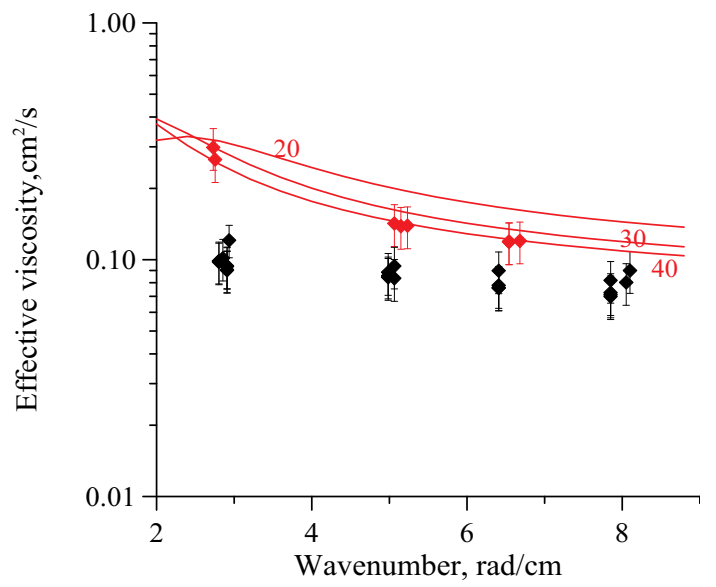

Fig. 7. Effective viscosity vs. wavenumber for EOF and COF (red and black symbols, respectively). Red curves - theoretical dependences of the "effective viscosity" for a fluid with the bulk viscosity of $0.1 \mathrm{~cm}^{2} / \mathrm{s}$ and film elasticities of 20,30 and $40 \mathrm{mN} / \mathrm{m}$ (from top to bottom). (For interpretation of the references to color in this figure legend, the reader is referred to the web version of this article.) assumption is supported by Fig. 2(b) which indicates that the surface tension coefficients are practically the same for both bulk oil emulsions and crude oil. This assumption seems to be realistic because the water particles in the surface microlayer of the oil-water emulsion should be covered with a film to minimize the total surface tension of the emulsion. Theoretically calculated "effective viscosity" dependences at different film elasticity values and at the oil emulsion bulk viscosity of $0.1 \mathrm{~cm}^{2} / \mathrm{s}$ are shown in Fig. 7. Comparison with the experimental values indicates that the elasticity of a thin film presumably covering the EOF surface varies in the range of about $20 \mathrm{mN} / \mathrm{m}$ and $30 \mathrm{mN} / \mathrm{m}$. From a physical point of view the existence of a quasi monomolecular film on the oil emulsion surface may not be very surprising. An oil emulsion is a heterogeneous fluid in which small droplets of pure water in the oil emulsion coexist on the free surface with small oil droplets on the free surface. Therefore, surfactants contained in the oil phase tend to cover the surface occupied by the droplets of pure water.

Concluding one can state that crude oil and oil emulsions can be approximately described as ordinary liquids characterized by constant viscosity and surface tension values, with an elastic film on the surface of oil emulsion.

\subsection{Wave damping in the presence of COF and EOF}

The wave damping coefficient as a function of thickness of COF and EOF, as mentioned in Section 3, demonstrates the occurrence of a maximum at some intermediate film thickness values. Since numerical calculations demonstrate similar peculiarities of the damping coefficient (see, Section 4), one can try to select film parameters in order to fit experiment and theory.

In Fig. 8 theoretical dependences of the damping coefficient and the effective surface tension for COF, calculated with the volume viscosity and surface tension coefficients for the upper surface and for the interface taken from experiment at the surface elasticity equal to zero are presented and compared with experiment for crude oil films. It is seen that theory both for the damping coefficient and for EST is roughly consistent with experiment for the interface elasticity values of 20 or 30 $\mathrm{mN} / \mathrm{m}$, although the maximum value at $\mathrm{E}=30 \mathrm{mN} / \mathrm{m}$ is larger than that in the experiment. One should note, however, that the location of the maximum and of the EST zigzag corresponds to smaller thicknesses, is up to half of the values obtained in the experiment. This discrepancy can be explained by rather large errors of estimation of the film thickness in experiment. The errors are associated with several effects. The first is the concentration of some amount of oil in the wall meniscus; this amount was estimated using a theory for the meniscus shape 


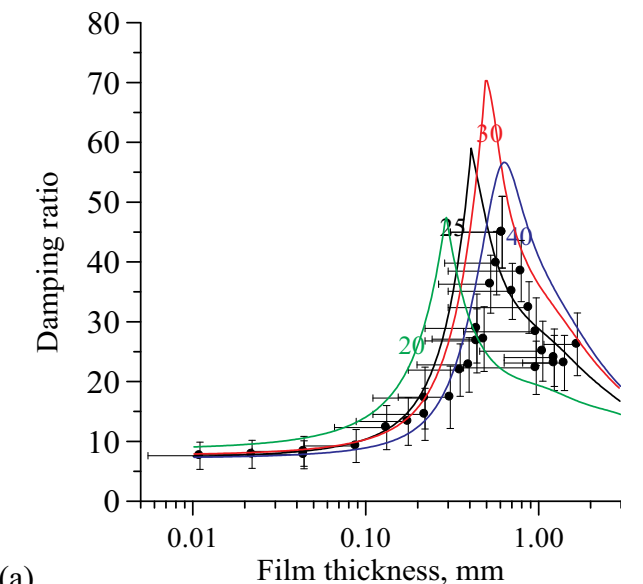

(a)

Film thickness, mm

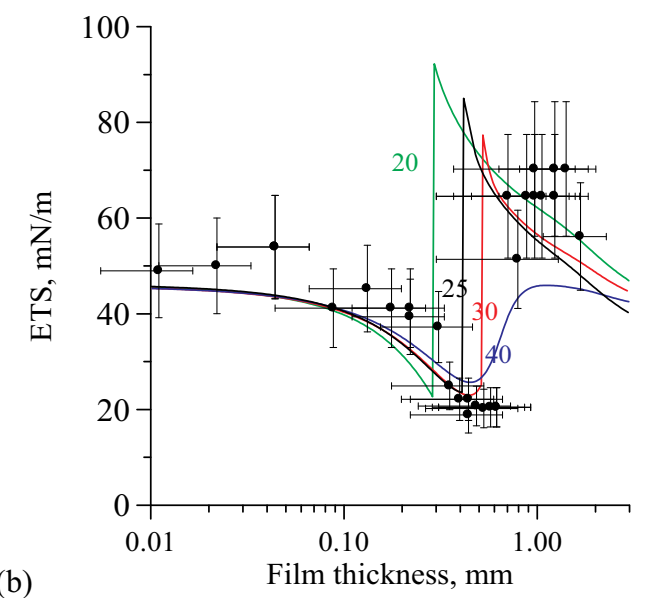

Fig. 8. Comparison between numerical calculations (curves) and experiment (points) for COF. The left panel is the relative wave damping, the right panel - the effective surface tension. The numbers on the curves of corresponding color denote the interface elasticity values in mN/m. Wave frequency $10 \mathrm{~Hz}, \nu_{+}=0.09 \mathrm{~cm}{ }^{2} / \mathrm{s}$, $\rho_{+}=0.85 \mathrm{~g} / \mathrm{cm}^{3}, \rho_{-}=1 \mathrm{~g} / \mathrm{cm}^{3}, \nu_{-}=0.0085 \mathrm{~cm}^{2} / \mathrm{s}, \sigma_{+}=30 \mathrm{mN} / \mathrm{m}, \sigma_{-}=20 \mathrm{mN} / \mathrm{m}, E_{+}=0$.

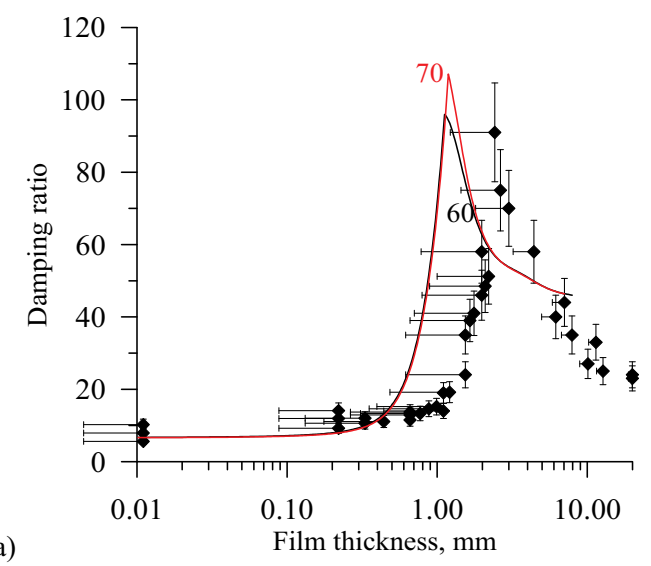

(b)

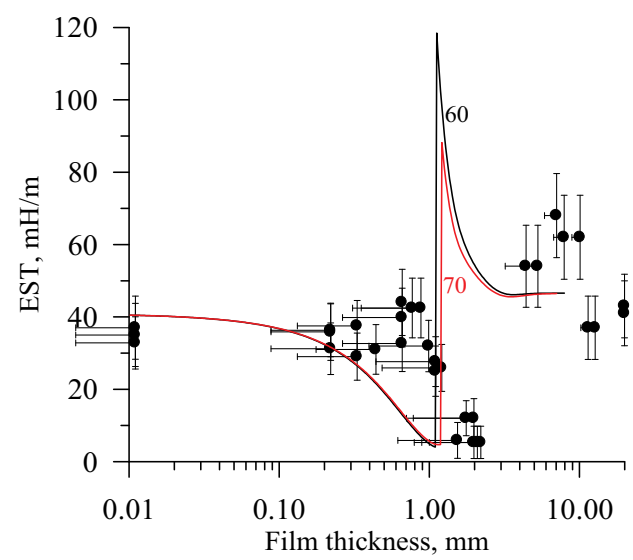

Fig. 9. Comparison between numerical calculations and experiment for oil emulsion films. The left panel is the relative wave damping, the right panel - the effective surface tension. The numbers on the curves denote the interface elasticity values. Wave frequency $10 \mathrm{~Hz}, \nu_{+}=0.1 \mathrm{~cm}^{2} / \mathrm{s}, \rho_{+}=0.85 \mathrm{~g} / \mathrm{cm}^{3}, \rho_{-}=1 \mathrm{~g} / \mathrm{cm}^{3}, \nu_{-}=$ $0.0085 \mathrm{~cm}^{2} / \mathrm{s}, \sigma_{+}=35 \mathrm{mN} / \mathrm{m}, \sigma_{-}=5 \mathrm{mN} / \mathrm{m}, E_{+}=30 \mathrm{mN} / \mathrm{m}$.

(see, Landau and Lifshitz, 1987) under the assumption of the zero edge angles. The overestimation of the film thickness due to the meniscus was obtained about $20 \%$. Another error is due to sticking of oil on the walls of the container. According to our observations the stack oil is contained in a layer of about $3-4 \mathrm{~mm}$ in height along the walls. If the thickness of the stuck oil is about $0.5 \mathrm{~mm}$, this gives us the overestimate of $\mathrm{COF} / \mathrm{EOF}$ of about $30 \%$ at the concentrations corresponding, for instance, to the damping maximum. The third error is due to film non uniformity. For mean film thicknesses more than about $0.3-0.5 \mathrm{~mm}$ some colored "patches" of larger thickness appear. According to our preliminary estimation the thickness variations can be about $50-100 \%$ of the mean thickness, or even larger. These estimates were based on measuring the oil amount added on water and the area of colored oil "patches". One should emphasize that the "patches" do not contribute strongly to the total damping coefficient unless the relative "patches" area is smaller than $50 \%$ or so. More in depth analysis of wave damping for the case of non-uniform oil films is to be presented elsewhere. Here we assume the error of thickness estimation for COF/OEF of about $100 \%$ in total, these error bars are depicted in Figs. 8 and 9. Note that the damping maximum in Figs. 8 and 9 occurs at film thickness comparable with the thickness of the gravity-capillary wave viscous boundary layer in oil film. From the physical point of view the boundary layers in oil film as well as in water beneath the film are strongly modified due to elasticities of the surface and interface similarly to what happens for the case of monomolecular elastic films on water. When the oil thickness becomes comparable to the thickness of the boundary layer the strong orbital velocity gradients in the film are formed thus resulting in strong dissipation and consequently in strong wave damping. Concerning the zigzag particularity of EST one should emphasize that the latter is not real, but effective surface tension, which is a function of the surface/interfacial tension coefficients and wave length and which can be clearly explained physically only in the limiting cases of thick and thin films (see, above). Mathematically, the damping maximum and the zigzag are related to the intersection of the imaginary parts of the dispersion curves for two wave solutions (modes) and these wave solutions will be discussed elsewhere in more detail.

Results of comparison between theory and experiment for EOF are shown in Fig. 9. From Fig. 9 one can conclude that at the surface elasticity of about $30 \mathrm{mN} / \mathrm{m}$ and the interface elasticity of $60-70 \mathrm{mN} / \mathrm{m}$ the damping maximum value is satisfactorily, although not perfect, consistent with theory, as well as the EST behavior. The main disagreement between theory and experiment however is in film thickness values that may be caused by the error of experiments (see, above). Namely, the film thickness corresponding to the damping maximum and the EST zigzag feature in experiment is about twice the theoretical thickness. This conclusion is similar to the case of COF.

Now we try to use the obtained characteristics of COF and EOF to interpret some experiments on radar probing of oil slicks using L-band UAVSAR (see, Minchew et al., 2012; Skrunes et al., 2016). To estimate radar contrasts - the radar cross section (RCS) damping ratio, we used a 


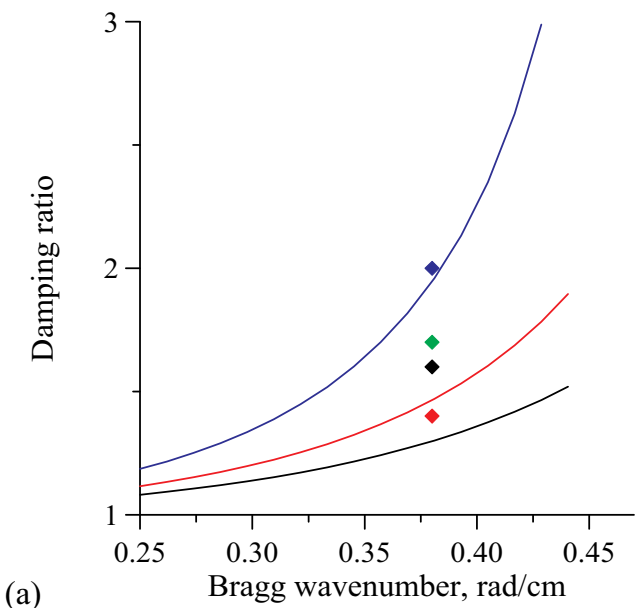

(a) $\begin{array}{lll}0.30 & 0.35 & 0.40 \\ \text { Bragg wavenumber, } \mathrm{rad} / \mathrm{cm}\end{array}$

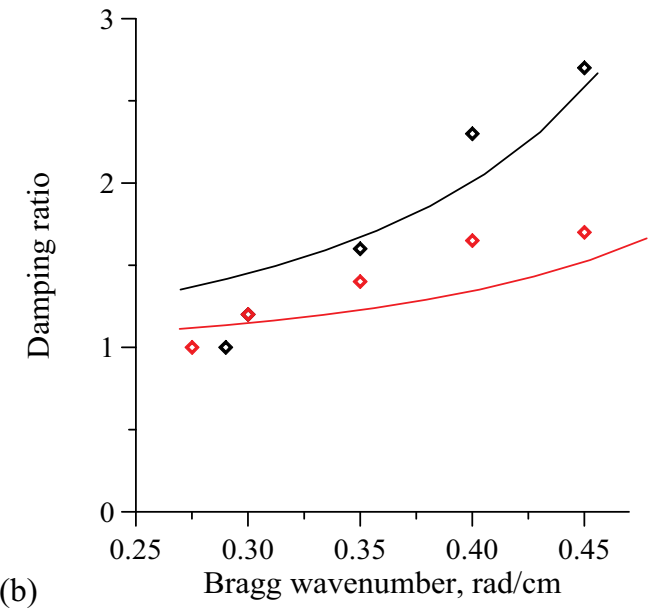

(b)

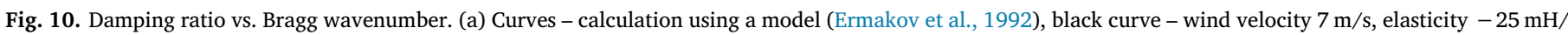

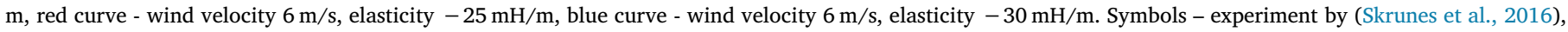

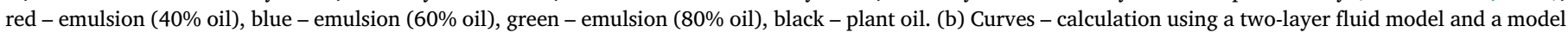

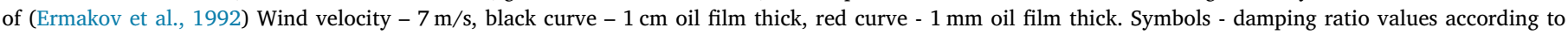
(Minchew et al., 2012). (For interpretation of the references to color in this figure legend, the reader is referred to the web version of this article.)

simple model of wind wave spectrum (Ermakov et al., 1992) in combination with a Bragg model of radar backscatter from the sea surface. Although the applicability of the Bragg model is not obvious, as it has been extensively discussed in the literature last years (see. e.g., Kudryavtsev et al., 2003, Ermakov et al., 2018), it can be approximately used for the cases of radar probing at the vertical (VV) polarization and at moderate incidence angles of microwave radiation (Ermakov et al., 2018; Sergievskaya et al., 2019). Then, according to Ermakov et al. (1992) the damping ratio can be expressed as

$\mathrm{DR}=\frac{\beta\left(k_{\mathrm{bragg}}, u *\right)-2 \gamma\left(k_{\mathrm{bragg}}, 0\right)}{\beta\left(k_{\mathrm{bragg}}, u *\right)-2 \gamma\left(k_{\mathrm{bragg}}, \text { film }\right)}$.

where $\beta\left(k_{\text {bragg }}, \mathrm{u} *\right)$ is the wind wave growth rate, $\mathrm{u} *$ is the friction velocity, $\gamma\left(k_{\text {bragg, }}\right.$, film $)$ is the wind wave damping coefficient, which depends on the surface/interface tension coefficients and film elasticities, oil thickness and viscosity.

Theoretical dependences of the damping ratio (Eq. (18)) for the characteristics of EOF and COF films which were retrieved above, for small thicknesses (less that $0.1 \mathrm{~mm}$ ) and for wind velocities ranged from $6 \mathrm{~m} / \mathrm{s}$ to $8 \mathrm{~m} / \mathrm{s}$ are shown in Fig. 10a as functions of Bragg wave number. Results of experiments by Skrunes et al. (2016) on radar probing of oil films using L-band UAVSAR at wind velocities presumably corresponding to the above range (in fact, wind velocity measurements in the experiment were not perfectly co-located) are shown in Fig. 10 for comparison. One should emphasize, that the film thickness in the experiments by Skrunes et al. (2016) was estimated as $\approx 0.1 \mathrm{~mm}$. One thus can conclude that the experiment is consistent with theory for thin films. Another comparison has been made with UAVSAR observations of the Deepwater Horizon oil spill of 2010 (Minchew et al., 2012) where presumably thick, but of unknown thickness, oil/oil emulsion films appeared on the sea surface. Model calculations for oil film thickness ranged from $1 \mathrm{~mm}$ to $1 \mathrm{~cm}$ indicated satisfactory agreements with experiment (see, Fig. 10b).

\section{Conclusions}

Damping of gravity-capillary waves and the effective surface tension on the surface covered with crude oil and oil emulsion films have been investigated in a wide range of film thickness, as well as on the bulk crude oil and oil emulsions using an original method of parametric wave excitation.

The kinematic viscosity and surface tension of crude oil retrieved from experiment are characterized as constant values, so that crude oil can be considered as ordinary fluid. The effective viscosity of emulsified oil films is obtained to depend on wavenumber and it is assumed that a thin surfactant film is formed on the surface of oil emulsions.

It is shown that a strong maximum of the wave damping coefficient occurs for films of crude oil and of oil emulsions floating on the water surface at some film thicknesses typically in the range from $0.1 \mathrm{~mm}$ to 1-2 mm. The effective surface tension demonstrates rather complicated zigzag dependence in this thickness range.

Numerical analysis of the dispersion relation for surface waves in the presence of viscoelastic films of finite thickness confirmed the main peculiarities of the wave damping coefficient and EST as functions of film thickness. From comparison between theory and experiment the main parameters of crude oil and oil emulsion films were retrieved, particularly viscosity and elasticity, which are mostly responsible for wave damping. In particular, crude oil films can be characterized by interface elasticity values of about $20-30 \mathrm{mN} / \mathrm{m}$ and negligible surface elasticity. The oil emulsion films should be described by both interface elasticity (of about $60-70 \mathrm{mN} / \mathrm{m}$ ) and the surface elasticity (of about 30 $\mathrm{mN} / \mathrm{m})$.

Detailed physical explanations of the peculiarities of wave damping due to oil/oil emulsion films will be done elsewhere.

\section{Author contributions}

I.S. analyzed the results and carried out numerical calculations, S.E analyzed the results and wrote the paper, T.L. carried out the experiments, G.J. participated in discussions of the results.

\section{Funding}

This research regarding experimental studies of wave damping due to COF in sections III was supported by the Russian Foundation for Basic Research (Project 16-05-00990), and the Chinese-Foreign Cooperation in key projects "The detection of oil spill and its ecological impact study" (No. 133337KYSB20160002) and the National Science Foundation of China (No. 41576032). The measurements of wave damping due to oil emulsions in Section 3, numerical calculations in Section 4, as well as retrieval of characteristics of EOF (Section 5) have been supported by the Russian Science Foundation (Project 18-1700224). 


\section{Declaration of Competing Interest}

None.

\section{References}

Adamson, A.W., 1982. Physical Chemistry of Surfaces, fourth edition. John Wiley \& Sons, Inc., Somerset, NJ (664 pp).

Alpers, W., Huehnerfuss, H., 1989. The damping of ocean waves by surface films: a new look at an old problem. J. Geophys. Res. 94 (C5), 6251-6266. https://doi.org/10. 1029/JC094iC05p06251.

Brekke, C., Solberg, A.H.S., 2005. Oil spill detection by satellite remote sensing. Remote Sens. Environ. 95 (1), 1-13. https://doi.org/10.1016/j.rse.2004.11.015.

Dysthe, K., Rovner, G., Rabin, Y., 1986. Damping of capillary waves by polymeric monolayers. Comparison with hydrodynamic theory. J. Phys. Chem. 90, 3894-3895.

Ermakov, S.A., Kijashko, S.V., 2006. Laboratory study of the damping of parametric ripples due to surfactant films. In: Gade, M., Huehnerfuss, H., Korenovski, G. (Eds.), Marine Surface Films. Springer, pp. 113-128.

Ermakov, S.A., Panchenko, A.R., Salashin, S.G., 1992. Film slicks on the sea surface and some mechanisms of their formation. Dynam. Atoms. Oceans. 16, 279-304. https:// doi.org/10.1016/0377-0265(92)90010-Q.

Ermakov, S.A., Kijashko, S.V., Konov, I.R., 1996. Determining the elasticity parameter of surfactant films from damping measurements of standing gravity-capillary waves. Izv. Atmos. Ocean. Phys. 32 (4), 544-547.

Ermakov, S.A., Sergievskaya, I.A., Zuikova, E.M., Goldblat, V.Yu., Shchegolkov, Yu.B. 2006. Wave tank study of phase velocities and damping of gravity-capillary wind waves in the presence of surface films. In: Marine surface films. Springer, pp. 129-143.

Ermakov, S.A., Sergievskaya, I.A., Gushchin, L.A., 2012. Damping of gravity-capillary waves in the presence of oil slicks according to data from laboratory and numerical experiment. Izv. Atmos. Ocean. Phys. 5 (48), 565-572.

Ermakov, S.A., Kapustin, I.A., Lazareva, T.N., Sergievskaya, I.A., Andriyanova, N.V., 2013. On the possibilities of radar probing of eutrophication zones in water reservoirs. Izv. Atmos. Ocean. Phys. 49 (3), 307-314.

Ermakov, S.A., Sergievskaya, I.A., Da Silva, J.C., Kapustin, I.A., Shomina, O.V., Kupaev, A.V., Molkov, A.A., 2018. Remote sensing of organic films on the water surface using dual co-polarized ship-based X-/C-/S-band radar and TerraSAR-X. Remote Sens. 10, 1097.

Fingas, Merv, 2018. The challenges of remotely measuring oil slick thickness. Remote Sens. 10, 319. https://doi.org/10.3390/rs10020319.

Fingas, M., Brown, Carl, 2015. In: Fingas, Merv (Ed.), Oil Spill Remote Sensing in Book: Handbook of Oil Spill Science and Technology. John Wiley \& Sons, pp. 313-356. https://doi.org/10.1002/9781118989982.ch12,2015. Chapter: 12.

Fingas, M., Fieldhouse, B., 2011. Studies on water-in-oil products from crude oils and petroleum products. Mar. Pollut. Bull. 64, 272-283.

Gade, M., Alpers, W., Hühnerfuss, H., Masuko, H., Kobayashi, T., 1998. Imaging of biogenic and anthropogenic ocean surface films by the multifrequency/multipolarization SIR-C/X-SAR. J. Geophys. Res. 103 (C4), 18851-18866. https://doi.org/ 10.1029/97JC01915.

Hansen, M., Kudryavtsev, V., Chapron, B., Brekke, C., Johannessen, J., 2016. Wave breaking in slicks: impacts on C-band quad-polarized SAR measurements. IEEE J. Sel. Top. Appl. https://doi.org/10.1109/JSTARS.2016.2587840.

Henderson, D.M., 1998. Effects of surfactants on faraday-wave dynamics. J. Fluid Mech. 365, 89-107.
Huehnerfuss, H., Lange, P., Walter, W., 1984. Wave damping by monomolecular surface films and their chemical structure. Pt.II: variation of the hydrophylic part of the film molecules including natural substances. J. Mar. Res. 42, 737-759.

Hühnerfuss, H., 1986. The Molecular Structure of the System Water/Monomolecular Surface Film and Its Influence on Water Wave Damping. Habilitationsschrift, University of Hamburg, Department of Chemistry, Hamburg, Germany, pp. 245.

Jenkins, A., Jakobs, S., 1997. Wave damping by a thin layer of viscous fluid. Phys. Fluids 9 (5), 1256-1264.

Kudryavtsev, V., Hauser, V., Caudal, D., Caudal, G., Chapron, B., 2003. A semiempirical model of the normalized radar cross-section of the sea surface: 1. Background model. J. Geophys. Res. 108 (C3), 8054. https://doi.org/10.1029/200IJC001003.

Landau, L.D., Lifshitz, E.M., 1987. Fluid Mechanics. Pergamon Press, Oxford.

Levich, V.G., 1962. Physicochemical Hydrodynamics. Prentice-Hall, Scripta Technica, Inc., Englewood, New York.

Lucassen-Reynders E.N., Lucassen J. Properties of capillary waves Adv. Colloid Interface Sci. 1969. V. 2. N 4. pp. 347-395.

Lucassen-Reynders, E.H., Lucassen, J., 1970. Properties of capillary waves. Adv. Colloid Interface Sci. 2, 347-395.

Migliaccio, M., Nunziata, F., Buono, A., 2015. SAR polarimetry for sea oil slick observation. Int. J. Remote Sens. 36 (12), 3243-3273. https://doi.org/10.1080/ 01431161.2015.1057301.

Miles, J.W., 1991. The capillary boundary layer for standing waves. J. Fluid Mech. 222, 197-205.

Minchew, B., Jones, C.E., Holt, B., 2012. Polarimetric analysis of backscatter from the Deepwater horizon oil spill using L-band synthetic aperture radar. IEEE Trans. Geosci. Remote Sens. 50 (10), 3812-3830.

Pogorzelski, S., Linde, B., Sliwinski, A., 1984. Capillary wave attenuation on a water surface coated with monolayers of oil-derivative substances. Acoust. Lett. 8 (1), 5-9.

Rajan, G.K., Henderson, D.M., 2017. Linear waves at a surfactant-contaminated interface separating two fluids: Dispersion and dissipation of capillary-gravity waves. Phys. Fluids 30 (7), 072104. https://doi.org/10.1063/1.5027709.

Scott, J.C., Stephens, R.W.B., 1972. Use of moire fringes in investigating surface wave propagation in monolayers of soluble polymers. J. Acoust. Soc. Am. 52 (pt2), 871-878 N3.

Scott, J.C., Thomas, N.H., 1999. Sea surface slicks - surface chemistry and hydrodynamics in radar remote sensing. In: Sajjadi, S.G., Thomas, N.H., Hunt, J.C.R. (Eds.), Windover-wave Couplings. Perspectives and Prospects. Clarendon Press, USA, New York, Oxford, 0-19-850192-7, pp. 221-229.

Sergievskaya, I.A., Ermakov, S.A., 2017. Damping of gravity-capillary waves on water surface covered with a visco-elastic film of finite thickness. Atmos. Ocean. Phys. 53, 650. https://doi.org/10.1134/S000143381706010X.

Sergievskaya, I., Ermakov, S., Lazareva, T., 2016. On damping of short gravity-capillary waves due to oil derivatives film on the water surface. In: Proc. SPIE - The International Society for Optical Engineering "Remote Sensing of the Ocean, Sea Ice, Coastal Waters, and Large Water Regions 2016" C, pp. 999903.

Sergievskaya, I., Ermakov, S., Lazareva, T., Lavrova, O., 2017. Damping of surface waves due to oil emulsions in application to ocean remote sensing. In: Proc. SPIE Vol. 10422, Remote Sensing of the Ocean, Sea Ice, Coastal Waters, and Large Water Regions 2017, pp. 104221H. https://doi.org/10.1117/12.2278575.

Sergievskaya, I.A., Ermakov, S.A., Ermoshkin, A.V., Kapustin, I.A., Molkov, A.A., Danilicheva, O.A., Shomina, O.V., 2019. Modulation of dual-polarized X-band radar backscatter due to long wind waves. Remote Sens. 11, 423.

Skrunes, S., Brekke, C., Jones, C.E., Holt, B., 2016. A multisensor comparison of experimental oil spills in polarimetric SAR for high wind conditions. IEEE J. Sel. Top. Appl. Earth Obs. Remote Sens. 9 (11), 4948-4961. 